\title{
Resolving the Unencoded Character Problem for Chinese Digital Libraries
}

\author{
Derming Juang, Jenq-Haur Wang, Chen-Yu Lai, Ching-Chun Hsieh, \\ Lee-Feng Chien, and Jan-Ming Ho \\ Institute of Information Science, Academia Sinica, Taiwan \\ \{derming, jhwang, lawrence, hsieh, Ifchien, hoho\}@iis.sinica.edu.tw
}

\begin{abstract}
Constructing a Chinese digital library, especially for a historical article archiving, is often bothered by the small character sets supported by the current computer systems. This paper is aimed at resolving the unencoded character problem with a practical and composite approach for Chinese digital libraries. The proposed approach consists of the glyph expression model, the glyph structure database, and supporting tools. With this approach, the following problems can be resolved. First, the extensibility of Chinese characters can be preserved. Second, it would be as easy to generate, input, display, and search unencoded characters as existing ones. Third, it is compatible with existing encoding schemes that most computers use.
\end{abstract}

This approach has been utilized by organizations and projects in various application domains including archeology, linguistics, ancient texts, calligraphy and paintings, and stone and bronze rubbings. For example, in Academia Sinica, a very large full-text database of ancient texts called Scripta Sinica has been created using this approach. The Union Catalog of National Digital Archives Project (NDAP) dealt with the unencoded characters encountered when merging the metadata of 12 different thematic domains from various organizations. Also, in Bronze Inscriptions Research Team (BIRT) of Academia Sinica, 3,459 Bronze Inscriptions were added, which is very helpful to the education and research in historic linguistics.

\section{Categories and Subject Descriptors}

H.3.7 [Information Storage and Retrieval]: Digital Libraries; H.3.5 [Information Storage and Retrieval]: Online Information Services.

\section{General Terms: Languages}

\section{Keywords}

Character Encoding, Unencoded Chinese Characters, Digital Library, Glyph Expression

\section{INTRODUCTION}

Missing or unencoded characters for a language present practical problems in digital library applications including character encoding, text input, font generation and display, document distribution and searching, and so on. The problems are particularly serious for large character sets, such as the Chinese character ${ }^{1}$ set, and other Han-related character sets. Constructing a Chinese digital library, especially for a historical article archiving, is often bothered by the small character sets supported by the current computer systems. This paper is aimed at resolving the unencoded character problem with a practical and composite approach for Chinese digital libraries.

In Chinese digital libraries, there's a fundamental problem in representing unencoded Chinese characters. The number of Chinese characters has been growing over time. This is due to the extensibility of Chinese characters which comes from the componential nature of characters. Moreover, the set of Chinese characters is not a closed finite set, and there's a lack of character standard.

The number of Chinese characters far exceeds the available coding space in computers. For example, the GB-2312 code standard contains 6,763 characters in Simplified Chinese, while the Big5 code standard contains 13,053 characters in Traditional Chinese. Even though the Unicode Standard [20] tries to solve the problem by expanding the coding space to a much larger space of 70,207 characters in Unicode 3.1, the fundamental problem still exists: there're always some Chinese characters unencoded. According to a statistics in a full-text database for Chinese ancient documents called Scripta Sinica [19], there are 22,780 distinct characters in a total of 200 millions characters of ancient texts such as Ershiwu shi ${ }^{2}$, Shisan jing ${ }^{3}, \ldots$ etc. Among these 22,780 characters, 9,727 (42.7\%) [11] cannot be encoded using Big5 code. Even in modern usage of Chinese characters, the problem still exists. For example, some special characters in person names and location names cannot be correctly displayed and input. Due to naming convention or personal preferences of characters, people can always coin new characters according to their needs. In fact, there are more than 18,000 unencoded Chinese characters in the person names of the

\footnotetext{
${ }^{1}$ In this paper, we use the term "Chinese characters" to represent the CJKV ideographs or Han-related characters (漢字) which are commonly referred to as Hanzi in Chinese, Kanji in Japanese, or Hanja in Korean.

2 Ershiwu shi 二十五史, The Twenty-five Dynastic Histories.

${ }^{3}$ Shisan jing 十三經, The Thirteen Confucian Classics.
} 
Household Registration in Taiwan. More than 10 new characters are being added per month.

Research of Chinese digital libraries started in the mid-1990s. In these digital libraries, various full-text databases of ancient and modern documents has been built. For example, China Digital Library Project (CDLP) $[13,24]$ in China began in year 2000 with a 5 -year timeframe building contents and infrastructures based on global standards. National Digital Archives Project (NDAP) [17] in Taiwan aims to promote and coordinate content digitization and preservation at leading museums, archives, universities, research institutes, and other content holders. During these efforts, four aspects of impact on these digital libraries were observed, including character encoding, text input, font generation and display, and document distribution and searching. First, in small-scale solutions, a "user-defined font area" in the coding space has been commonly adopted for users to specify new code sequences and the corresponding fonts for unencoded characters. However, the limited user space, the tedious font creation process, and the problem of code sequence ambiguity could make the digital library contents unable to be shared. Second, it would be very difficult to memorize and input the code sequences. Third, fonts have to be created by users and displayed when a user area code is detected. Finally, searching for such characters is difficult if not impossible. Therefore, a total solution for the unencoded Chinese character problem is required.

To resolve the unencoded character problem, there are three requirements in computer representation. First, the extensibility of Chinese characters has to be preserved. Second, it should be as easy to generate, input, display, and search unencoded characters as existing ones. Third, it must be compatible with existing encoding schemes that most computers use.

In this paper, a composite approach to the representation of Chinese characters is proposed. The proposed approach consists of the glyph expression model, the glyph structure database, and supporting tools. As of the writing of this paper, the glyph structure database contains 59,220 characters of Standard Scripts with 12,681 sets of variants. Other characters include 177 Oracle Bone Inscriptions (甲骨文) with 25 variants, 3,459 Bronze Inscriptions (金文) with 1,055 variants, 11,100 Small Seal Inscriptions (小管) with 1,081 variants, and 372 Chu Bamboo Slips Characters (楚系文字) with 73 variants. More characters are continuously added. The goal of this approach is to propose a complete solution that can fully express Chinese characters including existing ones, both ancient and modern, and those to be coined. All these characters can be input, displayed, distributed, processed, and even searched in exactly the same way as ordinary characters without introducing too much inconvenience to users.

This approach has been utilized in various applications. First, in Academia Sinica, a full-text database of ancient texts called Scripta Sinica [19] was created which contains a very large collection of historic documents such as Ershiwu shi, Shisan jing, ... etc. Second, the Union Catalog of NDAP [21] dealt with the problem encountered when merging the metadata of 12 different thematic domains from various organizations. Third, in Bronze Inscriptions Research Team (BIRT) of Academia Sinica, 3,459 Bronze Inscriptions were added, which is very helpful to the education and research in historic linguistics. More systems in museums, archives, and content holders are being built using the proposed approach.

\section{BACKGROUND AND RELATED WORK}

In order to make for a more comprehensive reading for people not familiar with Chinese characters, an introduction to the background knowledge about Chinese characters, glyphs, encoding standards, and digital library efforts is provided.

\subsection{Chinese Characters and Glyphs}

The Chinese writing system was originally pictographic, that is, words were represented by pictures that closely resembled the meaning of the word. For example, the picture for "sun" looked much like the sun. This pictographic writing eventually developed into the more complex ideographic writing that we are more familiar with. Chinese writing is one of the only contemporary writing systems that still prominently bear traces of its pictographic origins.

Around 1500BC, Chinese scripts started to appear in inscriptions. The first national standardization effort of Chinese scripts waas done in the realm of the First Qin Emperor (c. 259-210BC). Several lexicons were built since then. Around 121AD, in the Eastern Han Dynasty, Shuowenjiezi ${ }^{4}$ tried to analyze and classify all of the 10,516 characters at that time. In the $12^{\text {th }}$ century, Guangyun ${ }^{5}$ collected 25,126 characters. In 1716AD, Kangxi Dictionary ${ }^{6}$ contained 47,043 characters. The number of Chinese character was increasing over time.

Chinese characters are differentiated only by the meanings they carried [6]. A character may have many glyphs or shapes. Glyphs are differentiated by their structures or skeletons. For example, the characters 'sun' and 'moon' have different meanings. The glyph of 'moon' in Standard Scripts looks quite different from the same character in Bone Oracle Inscriptions. During the history of Chinese characters, many different forms have been derived, for example, Oracle Bone Inscriptions (甲骨文), Bronze Inscriptions (金文), Seal Inscriptions (䇡), Official Script (隸書), and Standard Script (楷書). The evolution of characters can help us trace the origin and better understand their meanings.

Glyphs do not deal with the actual outlook of the writing, but fonts do. A font is a collection of general design rules for a specific style of typefaces. For example, it's possible to specify the size, the broadness of strokes, the ratio of broadness of vertical and horizontal strokes, etc. For the same glyph, there are different styles of writing and calligraphic variants such as 宋 (Song), 隸 (Clerical Scripts), 行 (Running Scripts), 草 (Grass Scripts), etc..

Chinese characters are extensible or productive in the sense that new characters can be generated out of existing ones. It's fundamental since a finite set of basic ideographic components and composition operators can be used to express an unlimited set of unique Chinese characters. There are two levels of elements in Chinese characters: strokes and components.

\footnotetext{
4 Shuowenjiezi 說文解字, “Elucidations of the Signs and Explications of the Graphs,” compiled by Xu Shen (許慎), 100AD.

5 Guangyun 廣韻, an ancient Chinese rhyme dictionary.

${ }^{6}$ Kangxi Dictionary 康熙字典, a dictionary compiled by Zhang Yushu (張玉書) et. al., 1716AD.
} 
Strokes are the atomic element constituting the writing and calligraphic shape in Chinese characters. From a serial group of strokes, components can be formed, which are the basic compositional units for characters. There are two types of components: basic and compound. Basic components, also known as radicals, are the minimum fundamental components that will not be further decomposed. Compound components are intermediate elements consisting of several radicals. In order to uniquely represent a glyph, it's natural to decompose a glyph in terms of its radicals.

In 1972, Ni [18] analyzed 16 different compositional operators of Chinese glyphs and found that only three of them, namely horizontal composition, vertical composition and containing composition, are frequently used and can be assembled as an effective system for representing the structure of glyphs. After analyzing the 9,129 glyphs in [12], 496 radicals were obtained. Named as the NCTU Radical Set (a.k.a Chiao-Tung Root System), it was the earliest study of glyph structure in Taiwan.

This radical set is unique in that it was obtained by optimizing the total number of the radicals and the averaged number of radicals per glyph. In general, the less the number of radicals, the longer the decomposition of glyph. In this set, the weighted average number of radicals per glyph is only 1.9. After checking against the 49,905 characters of a Chinese lexicon ${ }^{7}, 48,713$ characters $(97.6 \%)$ can be expressed using this set. The remaining 1,192 characters are ancient scripts. More details of the statistics and optimization of the NCTU Radical Set can be found in $[9,12]$.

\subsection{Chinese Character Encoding Schemes}

In order to represent Chinese characters in computers, many different encoding schemes have been proposed to distinguish among characters. For example, each character can be uniquely assigned a 2-byte code in schemes such as GB-2312, Big5, ..., etc. However, the encoding standard for Chinese characters is still in lack. In China, the Simplified Chinese encoded in GB-2312 code standard contains 6,763 characters, while GBK code contains 20,902 characters. In Taiwan and Hong Kong, the Traditional Chinese in Big5 code standard contains 13,053 characters, while the CNS 11643 code standard contains 48,027 characters. The Unicode Standard 3.1 [20] tried to unify all CJKV ideographs with a set of 70,207 characters.

Since there are more than 65,536 unique Chinese characters, only the frequently used characters can be represented if each character is represented by one 2-byte code. In existing encoding standards such as GB-2312 and Big5, no extra information such as the structural evolution and variations of characters is encoded except the code sequence of glyphs, which has no particular meaning besides indexing. In the character-glyph model [20], the underlying model in Unicode, abstract characters with semantic meanings are differentiated from concrete glyphs, the visual shapes of characters. Only characters are encoded, not glyphs [3]. Characters from existing CJKV character sets must be merged, not separately encoded. Therefore, the Unihan database [10], the largest subset of the Unicode Standard, was introduced as the repository for the Unicode Consortium's knowledge regarding CJKV ideographs.

\footnotetext{
7 Zhongwen da cidian 中文大辭典, “Great Dictionary of the Chinese Language," edited by Zhang Qiyun, 40 vols., 1962-1968. Revised edition, 1973, 10 vols.
}

Different from the Unicode model, we adopt an analytic representation of characters, by breaking them down into pieces or components. This is similar to an ideographic composition scheme called Ideographic Description Sequence (IDS) [14, 15] later adopted by Unicode with a different set of operators and components. Recently, a character description language (CDL) [1] was also proposed to describe the characteristics of characters, which is more versatile than IDS.

\subsection{International Efforts in Chinese Digital Libraries}

Many international efforts dealt with the problems encountered when building Chinese digital libraries. Wittern $[22,23]$ introduced the problems encountered when dealing with digitization of Chinese Buddhist texts. CHANT project [4] in Hong Kong aimed to build an electronic database of all 1500BC to 600AD Chinese ancient texts. Richard Cook [2] discussed the character set issues relating to the computerization of an ancient Chinese lexicon Shuowenjiezi.

In China, CDLP (China Digital Library Project) [13, 24] was a 5 -year project starting from year 2000 building contents and infrastructures based on international standards. In Taiwan, NDAP (National Digital Archives Project) [17] started from 2002. Based on the experiences obtained from Digital Museum Project, Archives Digitization Project, and International Digital Library Cooperation Project, NDAP aims to digitize and preserve all kinds of documents across various organizations in different domains.

\subsection{A Brief History on Handling the Unencoded Characters}

Before the prevalence of personal computers, there has been some search on building a Chinese computer which is capable of inherently representing, encoding, inputting, and displaying Chinese characters. Since the number of Chinese characters far exceeds the alphabets in English, it's impractical to put each character in a separate key. In fact, in Chinese publishing industry, there was a "big" keyboard consisting of some 5,000 frequently used characters. A smaller version of some 500-key keyboard was also adopted.

The preliminary research on the problem of unencoded characters was based on the study in Chinese characters for computer use in National Chiao-Tung University (NCTU) $[12,18]$. The fundamental set of radicals and character composition operators was analyzed.

In 1984, the needs for the digitization and preservation of Chinese ancient texts grew stronger in Academia Sinica, and solutions on the related issues of Chinese character encoding and glyph rendering started to take shape $[5,7,8,11]$. Digitized ancient texts originally included Ershiwushi, which later evolved into Scripta Sinica [19] consisting of more than 200 million characters in total. In 1993, more than 9,700 unencoded characters have been collected. We started to focus on the solution on glyph expression model and the glyph database, which also evolved over the years.

Since 2002, NDAP [17] was started and the unencoded character problem became a critical issue when digitizing, organizing, and integrating more diverse texts and multimedia objects from various domains. Up to now, more than 100 databases from 12 thematic groups were integrated using the OAI-based [16] Union Catalog [21].

With growing popularity and usage of the Web, our PC-based solution was improved to accommodate the increasing needs for Web documents processing. Web-based intelligent character system 
was proposed to deal with the input, display, and search of unencoded characters in Web pages. A Web-based input method was used to compose unencoded characters. A browser plug-in module was designed to filter the Web pages, convert the glyph expressions into corresponding glyphs, and display them on the fly.

\section{GLYPH EXPRESSION AND GLYPH STRUCTURE DATABASE}

In the proposed approach, there are three main components, the glyph expression, the glyph structure database, and supporting tools.

\subsection{Representation of Chinese Glyphs}

Our glyph model is shown in Table 1. In this model, there are two parts: the representation of glyphs and the glyph structure expression. A formal representation of Chinese glyphs in Backus Naur Form is shown in Table 1.

Table 1. A formal representation of Chinese glyphs in Backus Naur Form.

$<$ character set $>::=\quad<$ glyph $>\mid<$ symbols $>$

$<$ symbols $>::=$ punctuation symbols, pronunciation symbols, and others

$$
\begin{aligned}
& <\text { glyph }>::=<\text { radical }>\mid<\text { component }>\text { | } \\
& <\text { glyph }><\text { operator }><\text { glyph }> \\
& <\text { component }>::=<\text { radical }>\mid \\
& <\text { component }><\text { operator }><\text { component }> \\
& <\text { operator }>::=\quad-\left.(\text { horizontal })\right|^{\wedge} \text { (vertical) } \mid @ \\
& \text { (containing) } \\
& <\text { radical }>::=492 \text { radicals }
\end{aligned}
$$

Mathematically speaking, the representation in Table 1 is productive in that the possible produced outcomes, such as glyphs and components are usually far more than we may accept. Whether the outcome is a legal item or not is up to our choice. Thus, this representation has the property of extensibility that just fit the requirement.

In this productive system, the structure of a glyph is expressed as an expression of radicals. Radical is a basic component which will not be decomposed further. Component is usually referred to the intermediate elements between a glyph and its radicals. An example of glyph structure decomposition is shown in Figure 1. In Figure 1, 㶓 and 戕 are components and also glyphs, while \#, 升, 戈, and 臣 are radicals, where 臣 is also a glyph.

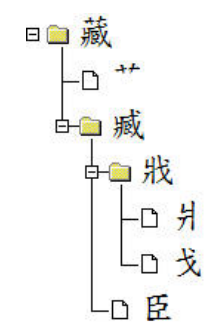

Figure 1. An illustration showing the glyph decomposition of a character “藏”。

Example sets of radicals and components are shown in Figure 2 and Figure 3, respectively.

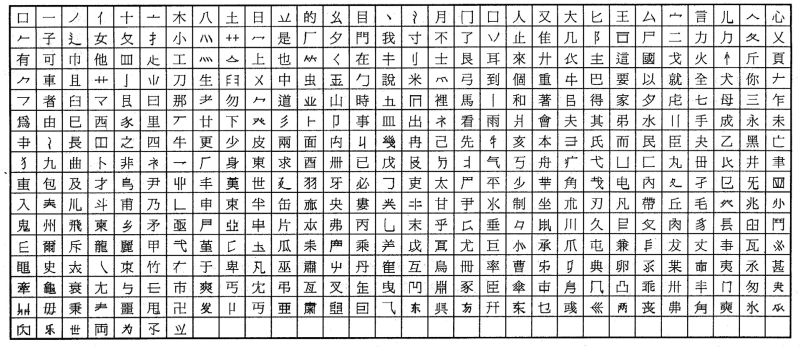

Figure 2. An example set of radicals.

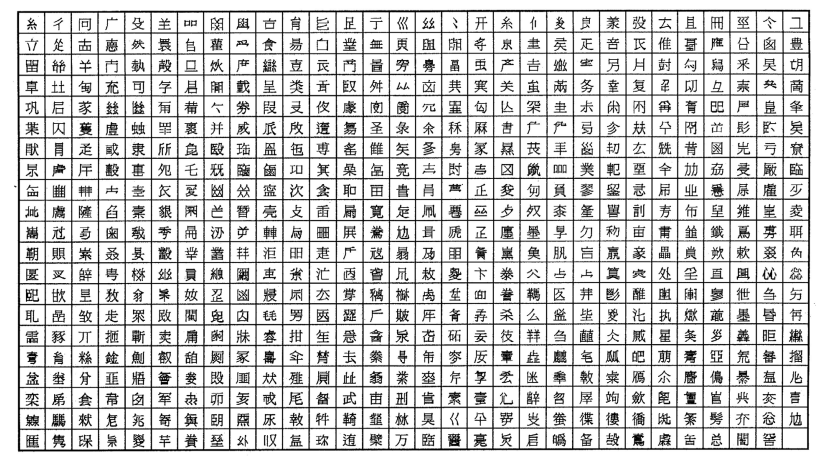

Figure 3. An example set of components.

\subsection{Glyph Structure Expression}

Glyph structure expression (or glyph expression for short) can be used to represent any existing character and those to be created in a user friendly way, which helps people memorize the code sequence very easily. Existing characters including ancient and modern characters can be looked up using the glyph expression. Ancient scripts such as Oracle Bone Inscriptions, Bronze Inscriptions, Seal Inscriptions and their evolutionary relationships can also be represented.

For any new unencoded character to be coined, they can also be created and represented in the same way as existing characters. All one need to know is how to write the glyph in its composing components.

Input methods like Changjie (倉頡) also uses the components to describe a character. In designing an input method, there is a tradeoff between the length of the code sequence representing a character and the number of conflicting characters with exactly the same code sequence. For users, it's important that a code sequence is efficient to type and easy to remember. The number of keystrokes (and thus the length of code sequence) should be minimized. For coding efficiency, on the other hand, the longer the code sequence, the more characters that can be represented, and also the less conflicting characters.

Different from an input method like Changjie, we are not concerned about the number of keystrokes. Instead, the extensibility of characters and the user convenience for looking up characters and creating new ones are the primary consideration. Glyph expressions can represent the most characters with no conflicting representations or ambiguity.

Let us explain how the structure of a glyph is expressed by examples. In Figure 1, the glyph ‘藏’ can be decomposed as: 


$$
\begin{aligned}
& \text { 藏 }=\text { サ 藏 } \\
& \text { 喊 }=\text { 践@臣 } \\
& \text { 戕 }=\text { 只 }- \text { 戈 }
\end{aligned}
$$

In Equation 1, these formulae are generally referred to as glyph expressions, in which “^”, "_“, and "@”" represent the operators of vertical, horizontal, and containing compositions, respectively. An expression consisting of only radicals can be obtained by successively decomposing components as follows:

$$
\begin{aligned}
\text { 藏 } & =\# \wedge \text { 㶓 } \\
& =+\wedge(\text { 戕@臣 }) \\
& =+\wedge((\text { 年 }- \text { 戈)@臣 })
\end{aligned}
$$

In Equation 2, the glyph “藏” is composed of 4 radicals. Note that except \#, which is encoded in Unicode, the other three radicals are included in Big5 code. Such glyph expression consisting solely of radicals is called a radical expression. Similarly, a glyph expression by components such as Equation 1 may be called a component expression. When all the operators are eliminated, Equation 2 becomes:

$$
\text { 藏 }=\text { 十年戈臣 }
$$

Equation 3 is called the radical sequence of the glyph '藏' showing the compositional order of radicals. Following the same naming thought, the formulae in Equation 1 after eliminating operators may be called component sequences which show the compositional order of components.

In general, any representation called "expression" refers to complete glyph structure information, while "sequence" does not. Each glyph has a unique glyph expression and hence this expression can be served as the identifier of that glyph. Although

"sequence" does not have complete structure information of glyph, it still has very high discriminating abilities among glyphs. For instance, in the 9,129 glyphs of [12], there are only 8 pairs of glyphs with exactly the same component sequence, such as (唄, 員) and (峰, 峯). All others can be uniquely identified by their component sequences.

According to our analysis, no character has exactly the same glyph expressions. This shows the uniqueness of glyph expressions with no conflicting characters. Since the representation is natural and intuitive in terms of character writing and composition, people can easily learn to use the glyph expression to represent characters in a short period of time. It's more convenient than user-defined font area since less laborious work needed for users.

\subsection{Chinese Glyph Structure Database}

Based on the idea of glyph expression model, it is easy to implement the glyph structure database (or glyph database for short), which contains the knowledge representation of glyph structure information for Chinese characters. For an unencoded character, we can look it up in the glyph database by its glyph expression or from the related characters by component lookup in the user interface of the glyph database as shown in Figure 4. We can easily input an unencoded character in a document. It can also help create characters including ancient ones. With the organization of glyph knowledge, the glyph database is more powerful than a font table.

This database has been extensively expanded and updated since 1993 [7, 11]. The current scale of the character set was enlarged from the set in [12] by including Simplified Chinese characters and the characters in Zhongwen da cidian and Hanyu da zidian ${ }^{8}$. As of the writing of this paper, the glyph structure database contains 59,220 characters of Standard Scripts with 12,681 sets of variants. Other characters include 177 Oracle Bone Inscriptions with 25 variants, 3,459 Bronze Inscriptions with 1,055 variants, 11,100 Small Seal Inscriptions with 1,081 variants, and 372 Chu Bamboo Slips Characters with 73 variants. These ancient characters are precious resources for Chinese studies and research, and more characters are being added.

Containing the rich knowledge of character composition, character evolution, and relational mappings among different forms of characters, the glyph database also provides an integrated user interface for exploring and searching within the database. As shown in Figure 4, both ancient and modern characters can be looked up by components, radicals, or glyph expressions within the glyph database. Then, the components and their structures can be displayed. In addition, the variants, the evolution, and the relations among various forms of the characters can be displayed. The related source information from related lexicons such as Shuowenjiezi can also be looked up.

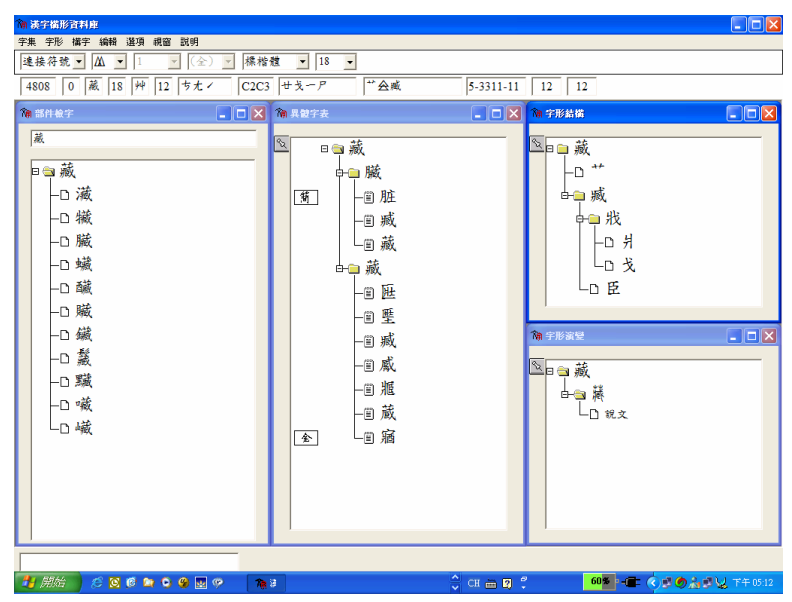

Figure 4. An illustration showing the integrated user interface for the glyph database.

\subsubsection{Optimization to the Glyph Database - the Kernel}

Set

There's a tradeoff between the user-friendliness of glyph expressions and the total number of components in the glyph database. The total number of components in the glyph database has to be minimized for coding space efficiency. On the other hand, to maintain an easy-to-use glyph expression, the average number of components per glyph has to be minimized. Therefore, there's a need for an optimization to the glyph database. This can be done by limiting the operators in a component expression to be one. In this case the number of components per glyph is usually 2, and occasionally 3 . After all one-operator expressions of a character set have been collected, a kernel set which has all necessary elements for one-operator expression for any one character/glyph in the original character set can be found. A pictorial presentation of the kernel set of our glyph database is shown in Figure 5. This kernel

\footnotetext{
${ }^{8}$ Hanyu da zidian 漢語大字典, Great Dictionary of Chinese Characters, edited by Hanyu da zidian weiyuanhui. Wuhan: Hubei cishu chubanshe and Sichuan cishu chubanshe, 1986.
} 
set includes 457 radicals (in which 269 are also characters and 188 non-character radicals), 629 components, and 1,044 kernel glyphs. There are 2,130 elements in total, where 1,313 are characters and the total frequency of usage is $62.60 \%$.

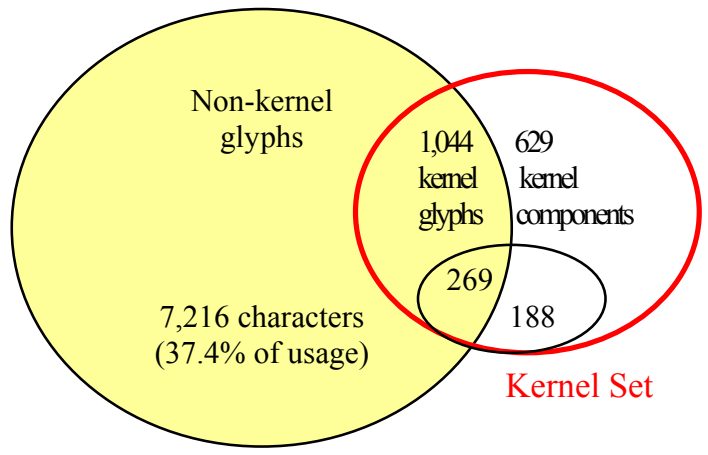

Figure 5. An illustration showing the kernel set of glyph database.

The kernel set in Figure 5 is not very efficient in terms of the total frequency of usage (only $62.60 \%$ ). This can be further optimized by including frequently used characters into the kernel set. A frequency distribution chart of our glyph database is shown in Figure 6. In Figure 6, it is obvious that including the 1,071 characters of the most frequently used category into the kernel will raise the total frequency of usage of the kernel to $97.8 \%$. The price paid is the increase of the numerical coding space to 3,201. It is not bad and can be afforded by any 2-byte encoding scheme.

Following the same thought, there are 5 possible levels of optimization to the kernel set as listed in Table 2. In Table 2, the fourth choice is recommended which includes all glyphs of the most frequently used and the frequently used categories. It contains 4,946 elements and the average code length is only 1.007 times of a 2-byte code.

Table 2. The weighted average code length at different levels of optimization in the character sets.

\begin{tabular}{|l|c|l|l|}
\hline \multicolumn{1}{|c|}{ Character Set } & $\begin{array}{c}\text { Number } \\
\text { of Codes }\end{array}$ & $\begin{array}{c}\text { The Weighted Average } \\
\text { Code Length }\end{array}$ & Entropy \\
\hline 1. Radicals & 457 & $1.9+1=2.9$ & 7.3038 \\
\hline 2. Kernel Set & 2,130 & $(37.4 * 3+62.6) \%=1.748$ & 8.6782 \\
\hline $\begin{array}{l}\text { 3. Kernel Set \& } \\
\text { Most Frequently } \\
\text { Used Characters }\end{array}$ & 3,201 & $(2.2 * 3+97.8) \%=1.044$ & \\
\hline $\begin{array}{l}\text { 4. Kernel Set \& } \\
\text { Frequently Used } \\
\text { Characters }\end{array}$ & 4,946 & $\begin{array}{l}\left(0.368 \mathrm{x}^{*} 3+99.632\right) \% \\
=1.007\end{array}$ & \\
\hline 5. The Whole Set & 9,346 & 1 & 9.1982 \\
\hline
\end{tabular}

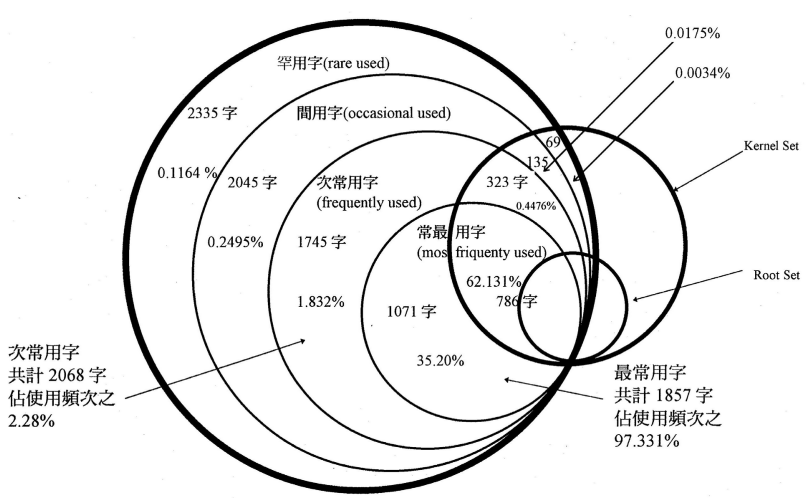

Figure 6. An illustration showing the kernel set and the frequency distribution chart.

\subsubsection{A Descriptive Method of Coding}

To save the coding space, a descriptive method of coding is adopted for characters not included in the kernel set. In ordinary numeric coding, each character is assigned a numeric code. This works for characters in the kernel set since it is a closed set with a relatively small $(2,130)$ total number of elements. They can be easily encoded into a 2-byte coding space. For characters not included in the kernel set, descriptive coding can be used where glyph expressions are used as the code sequences for characters instead of ordinary numeric codes. Descriptive code sequence does not occupy ordinary numerical coding space. Besides, descriptive coding is a productive system which is capable of representing additional newly created characters/glyphs to the existing character set.

Descriptive coding is compatible with existing codes. When 629 components and 188 non-character radicals of the kernel set as shown in Figure 5 are included into an existing encoding scheme such as GB, Big5, or Unicode, the descriptive capability can be provided. It can be further optimized by excluding some rarely used characters to save coding space, if necessary. If a kernel set for each country can be derived, then a CJK unified kernel can be formed, and hence a CJK unified descriptive code can be constructed.

\section{Supporting Tools}

In order to support the glyph expression and glyph database, tools for character encoding, text input \& display, font generation, document searching are required.

A scenario of the lifecycle of processing documents with unencoded characters is illustrated in Figure 7.

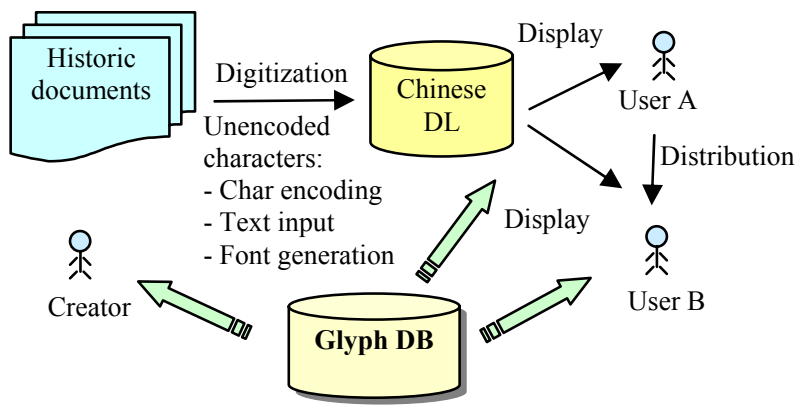

Figure 7. An illustration showing a scenario of the lifecycle of documents containing unencoded Chinese characters. 
As shown in Figure 7, the creator of a Chinese digital library has to first digitize the historic documents or antiques such as bronze and stone rubbings. In the digitization process, several issues arise for unencoded characters, for example: character encoding, text input, and font generation, among others. Second, for a user to see the digitized objects, a display tool has to be provided for the unencoded characters to be correctly shown. Third, when User A wants to distribute or disseminate the documents in Chinese digital libraries to User B, the information about the unencoded characters must be carried so that the same characters can be correctly input, displayed, and further utilized. Finally, applications such as document searching can then be supported.

To support the processing of documents containing unencoded Chinese characters with the glyph database, the necessary modules are illustrated in Figure 8.

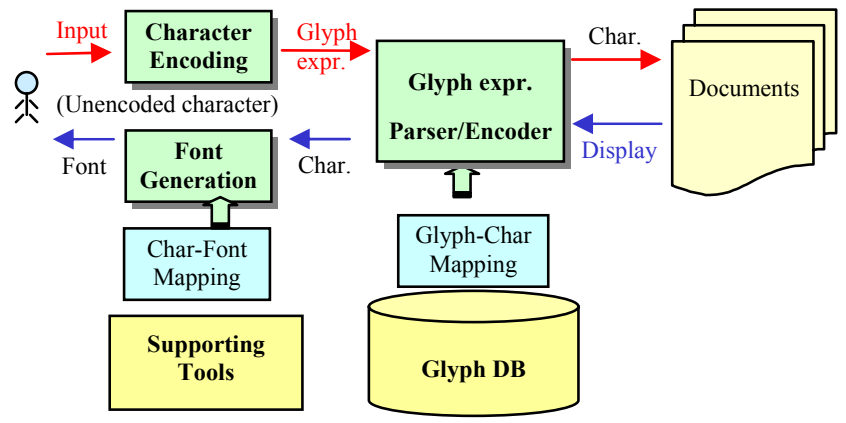

Figure 8. An illustration showing the components in supporting tools for unencoded Chinese characters.

\subsection{Tools for Character Encoding and Text Input}

As shown in Figure 8, there are two modules involved when inputting texts: character encoding and glyph expression encoder. In order to represent unencoded characters in ordinary documents, a character encoding module is needed to compose the glyph expression for unencoded characters. This can be obtained either by inputting glyph expressions directly with an unencoded character input method or by relevant glyph lookup with components or radicals from the user interface of glyph database. The user is not required to assign a new code sequence for the unencoded character. As mentioned earlier, since the glyph expression is unique for all glyphs, it can be used as the identifiers for characters.

Then, a glyph expression encoder module is needed to convert glyph expressions into character representation in documents. Markup tags are required to indicate the beginning and ending of the code sequence and glyph expression. Different markup tags might be needed for different kinds of documents such as Web pages and word processing files. Another possible way is to embed font images into the documents if further utilization of unencoded characters is not necessary.

There are several advantages to using glyph structure in encoding scheme. Firstly, the component set as well as the radical set of Chinese characters is a closed system. It will not expand indefinitely as the character set does. Although there are rare cases when they might need to be extended, they are far more manageable than that of character set.
The second advantage is that the glyph structure model is a productive system, as described earlier. This means it has the extensibility and flexibility of not changing the existing system over newly created characters. Thirdly, glyph structure is a kind of knowledge representation for Chinese characters. Characters can be represented by glyph expressions or component sequences. It not only facilitates character encoding and human reading, but also embeds more knowledge about characters into the existing encoding scheme for further applications in Chinese information processing.

\subsection{Tools for Font Generation and Display}

As shown in Figure 8, two modules are required when displaying texts: glyph expression parser and font generation. For an unencoded character to be correctly displayed, it has to be first converted from glyph expressions. Then, its associated font has to be generated and the corresponding glyph rendered. A resource locator indicating the location of the corresponding fonts will be encoded in texts.

For a document with glyph expressions, the glyph expression parser is needed to parse the expressions into corresponding characters. For example, after parsing a Web page with markup tags, the glyph expressions are extracted and the corresponding characters are identified. Then, a font generation module is used to get the corresponding fonts for characters in glyph database. For a document with font images, the corresponding font is directly retrieved and rendered. In fact, the glyph database serves as the index for glyph-character mapping and character-font mapping for supporting tools.

\subsection{Tools for Document Distribution and Searching}

Since the glyph expression is a unique representation of characters, it's natural to distribute the documents in glyph expressions instead of the converted form of glyph fonts or font images. No user-defined fonts are needed. Also, the embedded information about the characters includes the locators of the fonts in glyph database. Information exchange can be done in a more natural and smooth way in which unencoded character problem can be effectively resolved.

As long as the glyph expression is used as the identifier of characters, the unencoded characters in glyph expressions can be searched in the same way as normal characters. Searching of a character can be done by inputting glyph expressions, components, radicals, or relevant glyph lookup with the same input method as in text input.

\section{APPLICATIONS IN DIGITAL LIBRARIES AND DISCUSSION}

Since 1984, more and more applications in digitizing historic documents and archives had been conducted in Academia Sinica [7, $8,11]$. Currently, the glyph expression and glyph database have been utilized by organizations and projects in various areas including archeology, linguistics, ancient texts, calligraphy and paintings, and stone and bronze rubbings (金石拓片), to name a few.

For example, in Academia Sinica, a full-text database for Chinese ancient documents called Scripta Sinica [19] contains more than 200 million characters of ancient texts such as Ershiwu shi, Shisan jing, ... etc. More than 9,727 characters are unencoded [11] if the documents were to be represented in Big5 code. Only 427 of them 
cannot be directly represented by our approach. That is, 9,500 (more than $95.6 \%$ ) of them can be directly resolved without adding new radicals or components.

In NDAP [17], there are currently 9 public institutions participating in the project. These include nearly 100 different databases from libraries, museums, universities, and research institutes in 12 thematic groups such as geology, zoology, botany, anthropology, archives, calligraphy \& painting, and rare books. In order to integrate the metadata and the mappings among various databases from such diverse domains, an OAI-based [16] Union Catalog was developed [21]. All services in NDAP can share the same solution in the union catalog. Currently, 140,000 pieces from the Institute of History and Philology of Academia Sinica (IHP) ${ }^{9}$ and 10,000 pieces from Han Wooden Slips (漢代簡牘資料庫) are being integrated. The unencoded character problem was among the problems encountered. Glyph database was also utilized to resolve the problem.

In Bronze Inscriptions Research Team (BIRT, 金文工作室) under IHP, a Lexicon of Pre-Qin Bronze Inscriptions of Bamboo Scripts (先秦金文簡牘詞彙資料庫) were constructed where 3,435 characters are unencoded using Big5 code. Among them, 1,462 characters $(42.6 \%)$ can be directly represented using our approach. The remaining 1,973 unencoded characters together with 2,253 more characters collected from other references on Bronze Inscriptions were added into the glyph database.

As of this writing, the December 2004 version of the glyph database was announced and included in a CD-ROM. The database, tools, documents, papers, and related information are also available for download in the following website: http://www.sinica.edu.tw/ cdp/.

\section{Results}

As an example result, the original message of a short passage (in Bronze Inscriptions) scripted on a Bronze ritual vessel is shown in Figure 9.

(a)

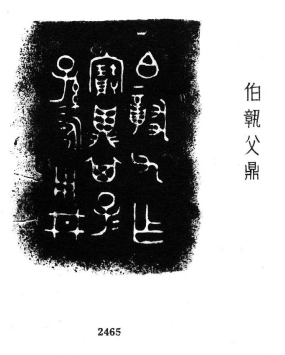

(b)

“白（伯）父乍（作）／寶鼎, 其子子／孫孫永用。〔井〕。”

(c)

“白（伯）璋父乍（作）／寶鼎，其子子／孫孫永用。〔井〕。”

Figure 9. An illustration showing the short passage scripted on a Bronze ritual vessel (伯璋父鼎): (a) the original image, (b) the

\footnotetext{
9 IHP (the Institute of History and Philology of Academia Sinica), http://www.ihp.sinica.edu.tw/.
}

\section{message displayed in Big5 code, and (c) the message in glyph expression.}

As shown in Figure 9, the unencoded character “璋“cannot be displayed in Big5, while it can be correctly displayed in glyph expression. More examples such as Mao-Kung Ting (毛公鼎) are available upon request.

\section{Discussion}

User-defined font area or private user area that most existing systems adopt is only a small-scale short-term solution to the unencoded character problem. It's neither scalable, nor portable.

Our approach identifies each character with its glyph expression, which is extensible to large-scale full-text Chinese databases or digital library applications. Also, the font is stored as a centralized resource on the Web, and only the resource locators are required to be embedded in documents. This approach helps resolve the portability issue.

The glyph expression model is extensible in that a finite set of components can be used to derive an open set of characters, including all possible forms from the past and those to be coined in the future. The current glyph database is built on top of existing Big5 coding space. The integration with other coding schemes such as Unicode is in progress.

\section{CONCLUSION}

In this paper, we proposed a glyph expression model for unencoded Chinese characters in digital libraries. The glyph expression model and the glyph database were introduced to resolve the unencdoed character problem. A scenario of dealing with documents containing unenecoded character was illustrated as well as the supporting tools for applying the model in character encoding, font generation, text display and input, and document dissemination. The statistics on current applications in Academia Sinica, NDAP program, and research teams on various domains also showed its potential in more applications in Chinese digital libraries.

\section{ACKNOWLEDGEMENTS}

This work was supported in part by the following grants: NSC 93-2752-E-001-001-PAE, 93-2422-H-001-0004 and 93-2213-E-001-025. We would like to express our heartily thanks to many researchers and scholars, such as Xue, Yong-Cheng (許永成), Jung, Bor-Sheng (鍾柏生) from IHP, Academia Sinica, Chi, Shiu-Sheng (季旭昇) from Department of Chinese, National Taiwan Normal University, and Wang, Ning (王寧) from Beijing Normal University. We have been cooperating with them for years for sharing the knowledge of Chinese Etymology (or Wen-Zi Xue, 文字學). The study and the system would not be possible without the efforts and supports from them.

\section{REFERENCES}

[1] Bishop, T. and Cook, R.S. A Specification for CDL Character Description Language. In Glyph and Typesetting Workshop, Kyoto, Japan, 2003.

[2] Cook, R.S. The Extreme of Typographic Complexity: Character Set Issues Relating to Computerization of the Eastern Han Chinese Lexicon Shuowenjiezi. In Proc. of the $18^{\text {th }}$ International Unicode Conference (IUC-18), Apr. 2001. 
[3] Cook, R.S. Typological Encoding of Chinese: Characters, Not Glyphs. In Proc. of $19^{\text {th }}$ International Unicode Conference (IUC-19), Sep. 2001.

[4] Ho, C. W. CHANT (CHinese ANcient Texts): a Comprehensive Database of All Ancient Chinese Texts up to 600 AD, Journal of Digital Information, Volume 3 Issue 2, Article No. 119, Aug. 2002.

[5] Hsieh, Ching-Chun. The Missing Character Problem in Electronic Ancient Texts (電子古籍中的缺字問題). In the First Conference on Chinese Etymology, Tianjin, Aug. 25-30, 1996. (in Chinese) http://www.sinica.edu.tw/ cdp/paper/1996/19960825_1.htm

[6] Hsieh, Ching-Chun. The Glyph and Encoding in Hanzi - On Redesigning Hanzi Interchange Code -- Part 1 (漢字的字形與 編碼). In International Conference on Hanzi Character Code and Database, Kyoto, Oct. 4, 1996. (in Chinese) http://www.sinica.edu.tw/ cdp/paper/1996/19961004_1.htm

[7] Hsieh, Ching-Chun (謝清俊). A Descriptive Method for Re-engineering Hanzi Information Interchange Codes - On Redesigning Hanzi Interchange Code -- Part 2. In International Conference on Hanzi Character Code and Database, Kyoto, Oct. 1996. http://www.sinica.edu.tw/ cdp/paper/1996/19961005_1.htm.

[8] Hsieh, Ching-Chun and Lin, Shih. A Survey of Full-text Data Bases and Related Techniques for Chinese Ancient Documents in Academia Sinica (中央研究院古籍全文資料庫的發展概 要), International Journal of Computational Linguistics and Chinese Language Processing, Vol. 2, No. 1, Feb. 1997. (in Chinese) http://rocling.iis.sinica.edu.tw/CLCLP/Vol2-1/a5.htm

[9] Hsieh, Ching-Chun. On the Formalization and Search of Glyphs in Chinese Ancient Texts (談古籍檢索的字形問題). In Conference on Rare Book and Information Technology, Taipei, Apr. 21, 1997. (in Chinese) http://www.sinica.edu.tw/ cdp/paper/1997/19970421_1.htm

[10] Jenkins, J.H. The Dao of Unihan. In Proc. of the 26th International Unicode Conference (IUC-26), Sep. 2004.

[11] Juang, Derming, Hsieh, Ching-Chun, and Lin, Shih. On Resolving the Missing Character Problem for Full-text Database for Chinese Ancient Texts in Academia Sinica (中央 㸴究院古籍全文資料庫解決缺字問題的方法). In the Second Cross-Strait Symposium on the Rectification of Ancient Texts, Beijing, May 11-13, 1998. (in Chinese) http://www.sinica.edu.tw/ cdp/paper/1998/19980511_1.htm
[12] Lin, S. (林樹). Research on the Fundamental Chinese Character Set for Computer Use (中文電腦基本用字㸴究), Technical Report, Department of Computer and Control Engineering, NCTU, March 1972. (in Chinese)

[13] Liu, W. The Development of Digital Collections and Metadata Applications in Chinese Libraries. In Proc. of International Symposium on Digital Libraries and Knowledge Communities in Networked Information Society (DLKC 2004), Japan, Mar. 2004.

[14] Lu, Q. (陸勤). The Ideographic Composition Scheme and Its Applications in Chinese Text Processing. In Proc. of the $18^{\text {th }}$ International Unicode Conference (IUC-18), Apr. 2001.

[15] Lu, Q., Chan, S., Li, Y., and Li, N. Decomposition for ISO/IEC 10646 Ideographic Characters. In the $3^{\text {rd }}$ Workshop on Asian Language Resources and International Standardization, COLING 2002, Taipei, 2002.

[16] OAI (Open Archives Initiative), http://www.openarchives.org/.

[17] NDAP, National Digital Archives Program, Academia Sinica (http://www.ndap.org.tw/)

[18] Ni, K (倪耿). 中國文字之結構模式及其分析, Master's Thesis, Institute of Electronics, NCTU, 1972. (in Chinese)

[19] Scripta Sinica, Hanji dianzi wenxian 漢籍電子文獻, Academia Sinica, http://www.sinica.edu.tw/ tdbproj/handy1/.

[20] The Unicode Consortium. The Unicode Standard, Version 4.0.1, defined by: The Unicode Standard, Version 4.0 (Reading, MA, Addison-Wesley, 2003. ISBN 0-321-18578-1), as amended by Unicode 4.0.1.

[21] Union Catalog of NDAP, http://catalog.ndap.org.tw/.

[22] Wittern, C. Chinese Buddhist texts for the new Millennium The Chinese Buddhist Electronic Text Association (CBETA) and its Digital Tripitaka. Journal of Digital Information, Volume 3, Issue 2, Article No. 123, Sep. 2002.

[23] Wittern, C. and App, U. IRIZ Kanji Base: A New Strategy for Dealing with Missing Chinese Characters. In EBTI (The Electronic Buddhist Text Initiative), Taipei, April 1996.

[24] Yang, G. and Zhang, T. The Development of the China Digital Library. Electronic Journal of Academic and Special Librarianship, Vol. 3, No. 3, 2002. 